\title{
The six-minute walk test in healthy children: reliability and validity
}

\author{
A.M. Li*, J. Yin*, C.C.W. Yu*, T. Tsang*, H.K. So*, E. Wong\#, D. Chan*, \\ E.K.L. Hon* and R. Sung*
}

ABSTRACT: The aim of this study was to assess the reliability and validity of the 6-min walk test (6MWT) in healthy children.

Chinese secondary school students were randomly recruited. They attended the current authors' unit on two occasions, separated by 2 weeks. Physical examination and standardised maximum incremental exercise testing on a treadmill were performed on the first visit. Spirometry and 6MWT were carried out on the second visit. A randomly selected subgroup was invited to return for repeat $6 \mathrm{MWT}$ at an interval of 2-4 weeks.

Seventy-eight subjects were recruited; however, four failed to achieve maximal effort on exercise test. The final group included 43 young females and the mean \pm SD age of the subjects was $14.2 \pm 1.2$ yrs. Physical examination was unremarkable in all cases. The mean \pm SD per cent predicted forced expiratory volume in one second was $91.4 \pm 10.2 \%$. Concurrent validity was demonstrated by good correlation between the 6-min walking distance and maximum oxygen uptake determined on the exercise treadmill. Test-retest reliability was undertaken in 52 subjects, and the intraclass correlation coefficient ( $95 \%$ confidence interval) was calculated as $0.94(0.89-0.96)$. In addition, Bland and Altman plots demonstrated a high degree of repeatability.

In healthy children, the 6-min walk test is a reliable and valid functional test for assessing exercise tolerance and endurance.

KEYWORDS: Children, six-minute walk, treadmill exercise

A $\mathrm{n}$ individual's response to exercise is an important clinical assessment tool, since it provides a composite assessment of their respiratory, cardiac and metabolic systems. The current gold standard for assessing a person's aerobic exercise response is the maximum incremental cardiopulmonary exercise test. However, most daily activities are performed at submaximal levels of exertion and, therefore, it has been proposed that submaximal functional tests are a better reflection of physical capability [1]. The ability to walk a set distance is a quick, easy and inexpensive way to assess physical function. It is also an important component of quality of life, since it reflects the capacity to undertake day-today activities. BALKE [2] developed a simple test for examining functional capacity: measuring the distance walked during a defined period of time. A 12-min performance test was then developed to evaluate the physical fitness of healthy individuals [3]. This test was subsequently modified for use in patients with chronic bronchitis [4]. In order to allow the test to be used in patients with respiratory diseases, for whom walking 12 min was too demanding, a shortened version, the 6-min walk, was developed, and found to perform equally as well [5]. A recent review of functional walking tests concluded that the 6-min walk test (6MWT) is easier to carry out, more acceptable and provides a better reflection of activities of daily living than other walk tests [1].

The 6MWT has been frequently used to measure outcomes before and after treatment in patients with moderate-to-severe heart and lung disease. It has also been used to measure functional status and for epidemiological research purposes. The distance covered in $6 \mathrm{~min}$ (6MWD) has been shown to accurately predict morbidity and mortality from cardiopulmonary diseases [6]. Previous studies involving children using the $6 \mathrm{MWT}$ have been performed in groups of diseased patients with physical dysfunction, but have not included healthy controls [7-9]. Therefore, the aim of this study was to establish the reliability and validity of the 6MWT performed in a cohort of healthy Chinese children.
AFFILIATIONS

*Dept of Paediatrics, and

\#Centre for Epidemiology \& Biostatistics, Prince of Wales Hospital, The Chinese University of Hong Kong, Shatin, Hong Kong.

CORRESPONDENCE

A.M. Li

Dept of Paediatrics

6th Floor

Clinical Sciences Building

Prince of Wales Hospital

Chinese University of Hong Kong Shatin

Hong Kong

Fax: 85226360020

E-mail: albertmli@cuhk.edu.hk

Received:

November 252004

Accepted after revision:

February 072005 


\section{METHODS}

\section{Study population}

The study was carried out over the summer (July-September) in 2004. The subjects were healthy students aged 12-16 yrs, who were randomly selected from local secondary schools. Each child underwent a thorough physical examination. Their weight and standing height were measured with a calibrated weighing scale and stadiometer using standard anthropometric methods [10]. Any subjects with underlying cardiopulmonary disease or conditions that could interfere with the walk test, or subjects who were taking any medications were excluded. All subjects underwent a standardised maximum incremental treadmill exercise test at their first visit and returned within 2 weeks for the $6 \mathrm{MWT}$. Spirometry was performed during the second visit before the subject underwent the walk test. A randomly selected group was asked to return 2-4 weeks after the initial 6MWT to have a supplementary walk test in order to assess its repeatability. Approval was obtained for the study from the Ethics Committee of the Chinese University of Hong Kong (Shatin, Hong Kong), and informed consent was obtained from parents and the subjects.

\section{Treadmill exercise test}

Aerobic capacity was assessed by running on an electromechanical treadmill (GE Marquette Medical Systems with centre ETT system; Advanced BioSystems, Hollywood, FL, USA). The treadmill was programmed according to the Bruce protocol. Breath-by-breath gas samples were collected via a comfortably fitted facemask (paediatric large size, 8950 series; Hans Rudolph, Kansas City, MS, USA) and analysed throughout the test by open-circuit calorimetry using the Medgraphics System CPX/DTM metabolic cart (Medical Graphics Corporation, St Paul, MN, USA). After a steady resting period of $1 \mathrm{~min}$, the subject was asked to exercise on the treadmill at a maintained speed. Care was taken to ensure complete safety and a physician was present during the whole procedure. Heart rate was monitored continuously during the test. Peak oxygen consumption $\left(V^{\prime} \mathrm{O}_{2}, \max \right)$, expressed as $\mathrm{L} \cdot \mathrm{min}^{-1}$, was determined when two of the following three conditions were reached: 1 ) respiratory exchange ratio $>1.0 ; 2$ ) heart rate $>85 \%$ of age-predicted maximum; or 3 ) the child was exhausted and refused to carry on despite strong verbal encouragement.

\section{Pulmonary assessment}

Spirometry (Spirolab II; Medical International Research, Rome, Italy), using standard techniques, was used to measure forced expiratory volume in one second (FEV1) and forced vital capacity. The best of three efforts was compared with local ageand sex-matched reference values [11]. This measurement was taken before the $6 \mathrm{MWT}$.

\section{Six-minute walk test}

The 6MWT was conducted according to standardised protocol [12]. A light meal was acceptable before the test, but the subject was told to avoid vigorous exercise within $2 \mathrm{~h}$ of beginning the test. No "warm-up" period before the test was allowed and the subject sat at rest in a chair, located near the starting position, for at least $10 \mathrm{~min}$ before the test started. During this time, pulse, oxygen saturation and blood pressure were measured. The subject was then instructed to walk up and down a measured corridor, covering as much ground as possible over a 6-min period. The test was self-paced and the patient was allowed to rest if desired, although the clock continued to run. The wording of encouragement during the testing was standardised ("keep going", "you are doing fine", " everything is going well") and given by the same person at set times during the test. The 6MWD was also recorded. Pulse and oxygen saturation were recorded at the end of the test. The $6 \mathrm{MWT}$ was performed by the same investigator who was unaware of the subjects' performance on the treadmill test.

\section{Statistical analysis}

Results are expressed as mean $\pm \mathrm{SD}$, unless otherwise indicated. Concurrent validity was assessed by performing Pearson's correlation on the association between $6 \mathrm{MWD}$ and $V^{\prime} \mathrm{O}_{2}$, max obtained from the treadmill exercise test. Inter-observer reliability of the 6MWT was analysed using Bland-Altman plots and by determination of the intraclass correlation coefficient. The level of significance was set at $5 \%$.

\section{RESULTS}

A total of 78 subjects were recruited; however, four failed to achieve maximal effort on the treadmill exercise test and were, therefore, excluded from the final analysis. The mean \pm SD age of the subjects was $14.2 \pm 1.2 \mathrm{yrs}$, and included 43 young females and 31 young males. None of the subjects was smokers and physical examination was unremarkable in all cases. Demographic data, lung function and characteristics of the $6 \mathrm{MWT}$ and treadmill test are shown in table 1.

Height $(\mathrm{r}=0.45, \mathrm{p}<0.0005), \mathrm{FEV}_{1}(\mathrm{r}=0.39, \mathrm{p}=0.001)$, heart rate at the end of $6 \mathrm{MWT}(\mathrm{r}=0.26, \mathrm{p}=0.024)$, heart rate difference at the end of 6MWT $(\mathrm{r}=0.42, \mathrm{p}<0.0005)$ and maximum heart rate achieved during the treadmill test $(\mathrm{r}=0.33, \mathrm{p}=0.004)$ were found to have significant correlation with $6 \mathrm{MWD}$. The $V^{\prime} \mathrm{O}_{2}, \max$ during the treadmill test was also found to have a significant correlation with $6 \mathrm{MWD}(\mathrm{r}=0.44, \mathrm{p}<0.0001)$. The scattergram showing the relationship between $6 \mathrm{MWD}$ and $V^{\prime} \mathrm{O}_{2}$, max is shown in figure 1.

\begin{tabular}{|c|c|c|c|}
\hline Characteristic & Whole group & Females & Males \\
\hline Subjects $n$ & 74 & 43 & 31 \\
\hline Age yrs & $14.2 \pm 1.1$ & $14.4 \pm 1.2$ & $13.9 \pm 1.1$ \\
\hline Weight kg & $48.6 \pm 8.5$ & $46.1 \pm 5.8$ & $52.1 \pm 10.4$ \\
\hline Height $\mathbf{c m}$ & $159.4 \pm 7.2$ & $156.7 \pm 4.7$ & $163.2 \pm 8.3$ \\
\hline Body mass index & $19.1 \pm 2.6$ & $18.7 \pm 2.0$ & $19.5 \pm 3.3$ \\
\hline FEV $1 \%$ pred & $91.4 \pm 10.2$ & $89.6 \pm 9.0$ & $93.9 \pm 11.3$ \\
\hline \multicolumn{4}{|l|}{ Heart rate beats $\cdot \mathrm{min}^{-1}$} \\
\hline Difference at end of 6MWT & $60.3 \pm 22.2$ & $58.8 \pm 24.3$ & $62.4 \pm 19.0$ \\
\hline At end of $6 \mathrm{MWT}$ & $147.8 \pm 18.7$ & $150.3 \pm 18.6$ & $144.3 \pm 18.5$ \\
\hline Max. during treadmill test & $196.4 \pm 9.6$ & $193.3 \pm 9.8$ & $200.7 \pm 7.5$ \\
\hline 6MWD m & $659.8 \pm 58.1$ & $637.4 \pm 38.6$ & $691.0 \pm 66.3$ \\
\hline$V^{\prime} \mathrm{O}_{2}, \max \mathrm{L} \cdot \mathrm{min}^{-1}$ & $1675.1 \pm 425.3$ & $1416.7 \pm 210.5$ & $2033.5 \pm 386.9$ \\
\hline
\end{tabular}

Data are presented as mean $\pm \mathrm{SD}$, unless otherwise stated. FEV 1 : forced expiratory volume in one second; \% pred: per cent predicted; 6MWT: 6-min walk test; max.: maximum; 6MWD: 6-min walk distance; $V^{\prime} \mathrm{O}_{2}$, max: maximum oxygen consumption at peak exercise on treadmill test. 


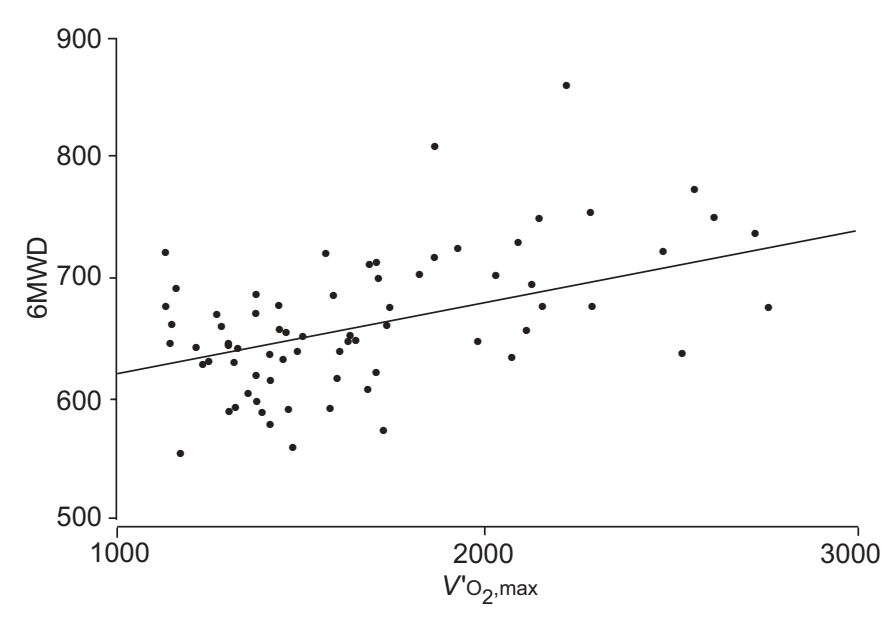

FIGURE 1. Relationship between 6-min walk distance (6MWD) and maximum oxygen consumption $\left(V^{\prime} \mathrm{O}_{2}, \max \right)$

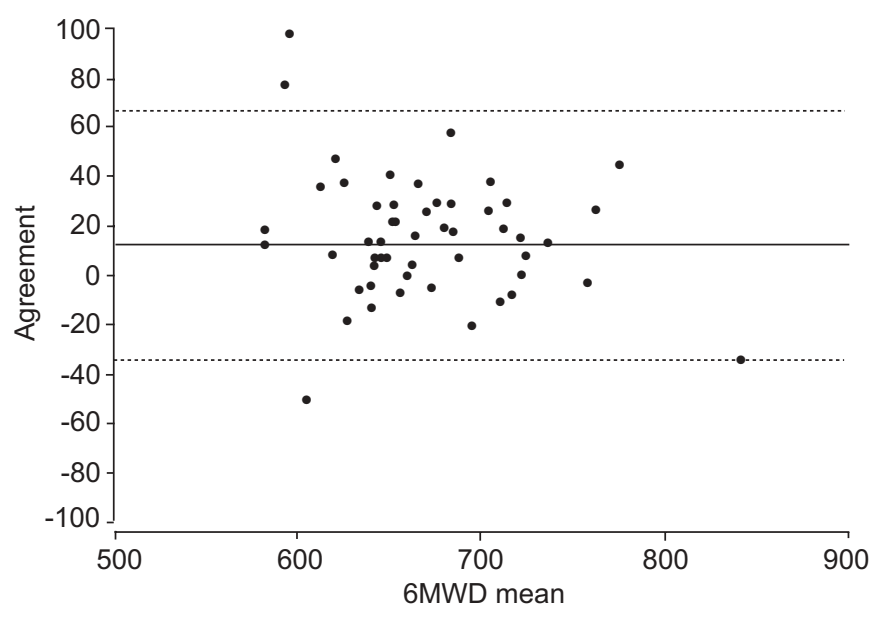

FIGURE 2. Bland-Altman plot of agreement of 6-min walk distance (6MWD) between two tests. The bias (mean difference between the two paired means) was $15 \mathrm{~m} \mathrm{(-)}$ and the limit of agreement (-----) was between -35 and $65 \mathrm{~m}$.

Test-retest reliability was evaluated in 52 subjects, 29 females and 23 males. The mean test-retest interval was 18 days, and ranged 14-29 days. There was no significant difference between the two walking distances reached $(662.21 \pm 55.1$ versus $677.23 \pm 50.8 \mathrm{~m} ; \mathrm{p}=0.56)$. Reliable consensus was observed for the $6 \mathrm{MWD}$. The Bland-Altman plots showed that the bias was $15 \mathrm{~m}$ and limits of agreement (2 SD) were -35 and $65 \mathrm{~m}$. The intraclass coefficient (95\% confidence interval) was 0.94 (0.89-0.96) (fig. 2).

\section{DISCUSSION}

In this study, it has been shown that the 6MWT in healthy children is reliable. There was no significant difference in the $6 \mathrm{MWD}$ reached at two time points separated by a mean of 18 days and the intraclass correlation coefficient was excellent. In addition, it was demonstrated that the test is valid, as a significant correlation was established between the 6MWD and $V^{\prime} \mathrm{O}_{2}$, max obtained during the treadmill exercise stress test.

The assessment of an individual's response to exercise is an important clinical tool, as it provides a global examination of the respiratory, cardiac and metabolic systems. The current gold standard for assessing the aerobic exercise response is the maximum incremental cardiopulmonary exercise test. This allows objective determination of the appropriate intensity needed to perform prolonged exercise, the quantification of factors limiting exercise and a definition of the underlying pathophysiological mechanisms [12]. However, as most daily activities are performed at submaximal levels of exertion, submaximal functional tests are more appropriate and allow a better reflection of physical capability. The 6MWT, which is easy to perform and cost-effective, has been proposed as the best indicator of functional capacity among all submaximal exercise tests [1]. Previous studies involving children using the $6 \mathrm{MWT}$ were performed in groups of diseased patients and did not include healthy controls [7-9]. GULMANS et al. [7] studied 23 children with cystic fibrosis (CF) and found the 6MWT to be valid and useful in assessing the exercise tolerance and endurance of children with mild-to-moderate symptoms of CF. They also found significant negative correlation with pulmonary hyperinflation. NiXON et al. [8] recruited 17 patients and performed $6 \mathrm{MWT}$ and the incremental exercise test as part of their pre-heart, lung or combined heart-lung transplantation work-up. They found the 6MWT to be a better indicator of functional capacity in severely ill children. This current study is the first to assess the reliability and validity of the 6MWT involving normal healthy children.

A significant correlation was found between the 6MWD and FEV1. This correlation has also been demonstrated in other studies involving patients with chronic pulmonary disease and, specifically, CF [7-9]. Height is another important determinant of the 6MWD. This is not surprising, as taller people have, in theory, a larger stride length and, thus, a greater $6 \mathrm{MWD}$. Young males are also found to have greater exercise capacity and 6MWD than young females, probably as a result of their greater muscle mass.

There are certain limitations to the present study. First, only 74 subjects were recruited. This sample size was inadequate for the establishment of reference equations for prediction of the total distance walked over $6 \mathrm{~min}$ for healthy children. Nevertheless, that was not the main objective of the study, as the aim was to demonstrated reliability and validity of 6MWT in healthy children. Secondly, this study involved only Chinese children aged 12-16 yrs. Similar assessment of the 6MWT would need to be repeated in other age groups and populations to ascertain its reliability and validity, as age, sex and height have all been shown to affect the 6MWD [12].

The American Thoracic Society Pulmonary Function Standards Committee recently developed guidelines for the 6-min walk test in clinical settings [12]. The 6-min walk test is easier to administer, a better reflection of daily activities and better tolerated than other walk tests [1]. It is a useful measure of functional capacity and, in this study, both reliability and validity of the test were demonstrated. 


\section{REFERENCES}

1 Solway S, Brooks D, Lacasse Y, Thomas S. A qualitative systemic overview of the measurement properties of functional walk tests used in the cardiorespiratory domain. Chest 2001; 119: 256-270.

2 Balke B. A simple field test for the assessment of physical fitness. Rep 63-6. Rep Civ Aeromed Res Inst US 1963; 53: 1-8.

3 Cooper $\mathrm{KH}$. A means of assessing maximal oxygen intake: correlation between field and treadmill testing. JAMA 1968; 203: 201-204.

4 Mcgavin CR, Gupta SP, Mchardy GRJ. Twelve-minute walking test for assessing disability in chronic bronchitis. BMJ 1976; 1: 822-823.

5 Butland RJA, Pang J, Gross ER, Woodcock AA, Geddes DM. Two-, six- and 12-minute walking tests in respiratory disease. BMJ 1982; 284: 1607-1608.

6 Enright PL. The six-minute walk test. Respir Care 2003; 48: 783-785.

7 Gulmans VAM, van Veldhoven NHMJ, de Meer K, Helders PJM. The six-minute walking test in children with cystic fibrosis: reliability and validity. Pediatr Pulmonol 1996; 22: 85-89.

8 Nixon PA, Joswiak ML, Fricker FJ. A six-minute walk test for assessing exercise tolerance in severely ill children. J Pediatr 1996; 129: 362-366.

9 Upton CJ, Tyrrell JC, Hiller EJ. Two minute walking distance in cystic fibrosis. Arch Dis Child 1988; 63: 1444-1448.

10 Tanner JM. Physical growth and development. In: Forfar JO, Arneil GC, eds. Textbook of Paediatrics. Edinburgh, Churchill Livingstone, 1984; pp. 304-305.

11 Ip MS, Karlberg EM, Karlberg JP, Luk KD, Leong JC. Lung function reference values in Chinese children and adolescents in Hong Kong. Am J Respir Crit Care Med 2000; 162: 424-429.

12 ATS statement: guidelines for the six-minute walk test. $A m$ J Respir Crit Care Med 2002; 166: 111-117.

13 Bland JM, Altman DG. Measuring agreement in method comparison studies. Stat Methods Med Res 1999; 8: 135-160. 